\title{
Acolhimento na Atenção Primária à Saúde: revisão integrativa
}

\author{
Reception in Primary Health Care: an integrative review
}

\author{
Larissa Rachel Palhares Coutinho', Ana Rita Barbieri², Mara Lisiane de Moraes dos Santos ${ }^{\mathbf{3}}$
}

RESUMO O presente estudo tem por objetivo analisar o conhecimento produzido sobre o acolhimento na Atenção Primária à Saúde, nos últimos oito anos. Trata-se de uma revisão integrativa, relacionada também ao acolhimento no âmbito da Estratégia Saúde da Família. Pode-se afirmar que o processo de acolhimento ainda não está totalmente sistematizado nos modelos de atenção à saúde, podendo ser esta uma justificativa para as dificuldades apresentadas tanto por profissionais quanto por usuários. São necessários estudos com novas abordagens ou estratégias para a sistematização do acolhimento nas unidades de Atenção Primária à Saúde, e verificar se estas têm realmente impacto na qualidade dos serviços e na satisfação dos usuários.

PALAVRAS-CHAVE Acolhimento; Atenção à saúde; Atenção Primária à Saúde; Estratégia Saúde da Família.

ABSTRACT The present study aims to analyze the knowledge produced about the reception in the Primary Health Care, in the last eight years. It is about an integrative review, also related to the reception within the Family Health Strategy. It can be stated that the reception process is not yet fully systematized in the models of health care, and this may be a justification for the difficulties presented by both professionals and users. There are necessary studies with new approaches or strategies for the systematizing of the reception in units of Primary Health Care, and verify if these actually have an impact on the quality of services and in the users satisfaction.

KEYWORDS User embracement; Health care (Public Health); Primary Health Care; Family Health Strategy.

\footnotetext{
1 Universidade Federal do Mato Grosso do Sul (UFMS) - Campo Grande (MS), Brasil. larissarachel@gmail.com

2 Universidade Federal do Mato Grosso do Sul (UFMS) - Campo Grande (MS), Brasil. ana.barbieri@ufms.br

3 Universidade Federal do Mato Grosso do Sul (UFMS) - Campo Grande (MS), Brasil. maralisi@globo.com
} 


\section{Introdução}

Um dos eixos marcantes a respeito dos cuidados primários em saúde surgiu em 1978 com a Declaração de Alma-Ata, na qual os cuidados primários foram situados como estratégia principal do sistema de saúde de um país (WHO, 2008). A atenção primária é uma abordagem que forma a base e determina o trabalho dos demais níveis de atenção à saúde. É a atenção que organiza e racionaliza o uso dos recursos, tanto básicos como especializados, direcionados para a promoção, a manutenção e a melhora da saúde (STARFIELD, 2002).

Tanto em países desenvolvidos quanto em menos desenvolvidos, é unanimidade que a Atenção Primária à Saúde (APS) seja o modelo-chave de um sistema de saúde eficaz (OPAS, 2007). Quando os sistemas de saúde de um país estão organizados com base na APS, apresentam melhores possibilidades de desempenho, devido principalmente à acessibilidade, à integralidade e à própria organização e otimização dos recursos (LIMA, 2011), além de apresentarem melhores resultados em indicadores de saúde, como redução da mortalidade, diminuição dos custos da atenção, maior acesso aos serviços, redução das internações e atendimentos de urgência (MENDES, 2013).

No Brasil, o termo utilizado como equivalente à APS é a Atenção Básica (AB). Estes termos são associados às noções de vinculação e responsabilização, acessibilidade, atenção abrangente e integral, resolutividade e protagonismo na gestão do cuidado (BRASIL, 2011).

O modelo utilizado no Brasil, orientado na APS, é a Estratégia Saúde da Família (ESF), que teve início em 1994 e foi idealizado, inicialmente, como uma ferramenta de extensão da cobertura assistencial. Atualmente, se configura como o maior programa assistencial no País e é considerado como um eixo estratégico reorganizador do Sistema Único de Saúde (SUS), carregando enorme potencial para estruturar de forma consistente a APS no Brasil. Tem sido apontado, por sua extensão e cobertura, como um modelo a ser seguido (ABRAHÃO, 2007; MENDES, 2013).

O Ministério da Saúde tem lançado mão de estratégias para adequar os conhecimentos às novas propostas de estruturação da atenção primária. Dentre elas, destaca-se a Política Nacional de Humanização (PNH). Esta política atua a partir de orientações éticas, clínicas e políticas, que se traduzem em determinados arranjos de trabalho. Dentre as estratégias que norteiam esta política, evidencia-se o acolhimento (BRASIL, 2006).

$\mathrm{O}$ acolhimento surgiu a partir das discussões sobre a reorientação da atenção à saúde, sendo elemento fundamental para a reorganização da assistência em diversos serviços de saúde, direcionando a modificação do modelo tecno-assistencial. É um dispositivo que está inserido na Política de Humanização do Ministério da Saúde (HumanizaSUS), e que vai além da recepção ao usuário, pois considera toda a situação da atenção a partir da entrada deste no sistema. Acolher significa humanizar o atendimento (HENNINGTON, 2005).

O acolhimento possibilita uma reflexão acerca dos processos de trabalho em saúde, pois estabelece uma relação concreta e de confiança entre o usuário e o profissional ou a equipe, estando diretamente orientado pelos princípios do SUS (BRASIL, 2010), podendo atender às demandas da sociedade e estabelecer relação com os outros serviços de saúde, de maneira regionalizada e hierarquizada. É um recurso destinado a apoiar a qualificação do sistema de saúde, pois possibilita ao usuário o acesso a um cuidado justo, ampliado e integral, a partir do reconhecimento de que esse acesso é um direito humano fundamental (CARVALHO ET AL., 2008; BARALDI; SOUTO, 2011).

O acolhimento facilita, dinamiza e organiza o trabalho de forma a auxiliar os profissionais a atingirem as metas dos programas, a melhorarem o trabalho e executarem um 
bom atendimento, predispondo a resolutividade do problema (CARDOSO ET AL., 2009).

Aponta-se o acolhimento como diretriz operacional fundamental do modelo assistencial proposto pelo SUS, a fim de garantir não só a acessibilidade universal, mas também a qualificação das relações, na qual escuta e atenção às necessidades são fundamentais ao processo para que o serviço ofereça uma resposta resolutiva às demandas dos usuários (BREHMER; VERDI, 2010).

O acolhimento é um mecanismo primordial para a APS, entretanto, somente recebeu destaque nos processos de trabalho das Equipes de Saúde da Família em um passado relativamente recente. Assim, são necessários estudos avaliativos sobre a incorporação do acolhimento nos serviços de APS. E é diante desse contexto que o presente estudo propõe, como objetivo, a análise do conhecimento produzido sobre o acolhimento na APS nos últimos oito anos.

\section{Métodos}

Trata-se de uma revisão integrativa, relacionada ao acolhimento nos âmbitos da APS e da ESF. Optou-se por esta modalidade de pesquisa por se tratar de uma ferramenta que proporciona uma síntese do conhecimento já produzido e fornece subsídios para a melhoria da assistência à saúde (MENDES; SILVEIRA; GALVÃO, 2008).

Para a elaboração deste estudo, foram seguidas as seguintes etapas: (1) identificação do tema e elaboração da questão norteadora; (2) busca ou amostragem na literatura; (3) coleta de dados; (4) análise crítica dos estudos incluídos; (5) interpretação dos resultados; e (6) apresentação da revisão/síntese do conhecimento (SOUZA; SILVA; CARVALHO, 2010).

Para a primeira etapa, estabeleceu-se a seguinte questão norteadora: Como está a construção do conhecimento sobre o acolhimento na APS e na ESF?
Realizou-se uma busca na literatura científica, utilizando-se dos seguintes critérios de inclusão: textos completos, com livre acesso a bases de dados nos idiomas português, inglês e/ou espanhol, publicados entre janeiro de 2007 e maio de 2014; e estudos que abordem acolhimento na atenção primária e saúde da família. Optou-se por esta faixa temporal por ter-se identificado que as principais publicações sobre acolhimento ocorreram a partir de 2006, após a ‘normatização’ da HumanizaSUS.

Foram utilizadas as seguintes bases de dados nacionais e internacionais: a Literatura Latino-Americana e do Caribe em Ciências da Saúde (Lilacs); a Bibliografia Brasileira de Enfermagem (BDENF); e a biblioteca eletrônica Scientific Eletronic Library Online (SciELO). Não foram incluídos editoriais, teses, dissertações e/ou estudos que não abordam temática relevante ao alcance do objetivo da revisão.

Para a seleção dos artigos foram utilizados os indexadores contidos dos Descritores em Ciências da Saúde (DeCS), contemplando os seguintes termos, nos idiomas português, inglês e espanhol: 'Acolhimento'; 'Atenção à Saúde'; 'Atenção Primária à Saúde', 'Estratégia Saúde da Família'.

Os dados coletados foram organizados e agrupados nas categorias temáticas que configuram o escopo central deste estudo. Para a análise dos dados foi realizado o cálculo de frequência simples, a fim de descrever a caracterização dos artigos encontrados. Após esta etapa, foram realizadas a leitura crítica e a discussão dos artigos selecionados.

\section{Resultados e discussão}

A busca resultou em 115 artigos e, a partir da análise crítica dos resumos, foram selecionados 30 artigos que atendiam aos objetivos propostos. A maioria das publicações se deu entre 2008 e 2010; nota-se o baixo índice de publicação sobre o tema nos dois últimos anos, conforme evidencia a tabela 1. 
Tabela 1. Distribuição dos artigos sobre acolhimento na Atenção Primária à Saúde publicados entre 2007 e 2014, em base de dados $(n=30)$

\begin{tabular}{lll}
\hline Ano & № & $\%$ \\
\hline 2007 & 03 & 10,0 \\
2008 & 06 & 20,0 \\
2009 & 06 & 20,0 \\
2010 & 06 & 20,0 \\
2011 & 04 & 13,4 \\
2012 & 03 & 10,0 \\
2013 & 01 & 3,3 \\
2014 & 01 & 3,3 \\
\hline
\end{tabular}

Fonte: Elaboração própria

Quanto ao tipo de estudo, 24 eram de natureza qualitativa (80\%) e 6 de caráter quantitativo (20\%).

Após leitura crítica e sistematização dos dados, puderam-se evidenciar três grupos de discussão: Processo de implantação do acolhimento; Acolhimento sob a ótica dos profissionais; e Acolhimento sob a ótica dos usuários.

\section{Processo de implantação do acolhimento}

Para este tópico, foram avaliados 14 artigos (42\%), onde foram discutidos aspectos referentes ao processo de implantação do acolhimento, em suas diretrizes conceituais e técnicas.

Houve duas abordagens nos textos avaliados: experiências bem-sucedidas quanto à implantação do acolhimento; e avaliações onde houve incongruências sobre como o acolhimento é compreendido e realizado. Podem-se observar dois modelos de atenção à saúde a partir do acolhimento: o primeiro, que é mais pontual e baseado na demanda espontânea, e respeita a ordem de chegada; e o segundo, baseado em um cuidado integral, longitudinal e interprofissional.

O acolhimento é compreendido como método de escuta qualificada, de forma a oferecer respostas, porém, alguns estudos demonstraram que, na prática, o acolhimento se restringe a atendimentos pontuais e a ações de atendimento às urgências (TAKEMOTO; SILVA, 2007; OLIVEIRA; TUNIN; SILVA, 2008; BREHMER; VERDI, 2010). Observa-se, então, limitação do acesso e atenção centrada na queixa-conduta.

Em contrapartida, no estudo de Cardoso et al. (2009), o acolhimento não foi vivenciado em momentos específicos do processo de trabalho, mas vivido integralmente no contexto das diferentes intervenções. Nota-se uma ambiguidade nos diferentes modelos organizacionais de operacionalização do acolhimento nas unidades de atenção primária.

Quanto aos lugares onde era feito o acolhimento, foi referido que se tratavam de diferentes espaços, tais como: recepção; sala de espera, de atendimento ou de vacina; locais de consulta de enfermagem; almoxarifado; portas de entrada da unidade; ou, ainda, nos ambientes de atividades extramuros e/ ou visitas domiciliares (CARDOSO ET AL., 2009). Esta questão de ambiência das unidades está pautada nas diretrizes da $\mathrm{PNH}$.

A visita domiciliar foi mencionada como um dispositivo de acolhimento que propicia, entre outros aspectos, um melhor conhecimento dos usuários e sua inserção na comunidade, facilitando o vínculo (SANTOS ET AL., 2013) e explicitando a importância da visita domiciliar no processo de acolhimento. 
Como obstáculos para a realização do acolhimento nos serviços, destacaram-se: limites estruturais, elencados pela própria estrutura física inadequada das unidades e pela escassez de profissionais; algumas questões éticas, como falta de privacidade e sigilo nas interações profissional-usuário; e falta de postura de escuta e comprometimento. $\mathrm{O}$ acolhimento é reprimido pela ausência de condições básicas de recebimento dos usuários, desrespeitando o seu bem privado e as suas necessidades individuais (MATUMOTO ET AL. 2009; BREHMER; VERDI, 2010; OLIVEIRA ET AL., 2010).

Brehmer e Verdi (2010) ainda relacionam que a (des)organização do número de profissionais apresenta um reflexo negativo, tanto para os usuários - que não recebem um atendimento de real efeito, quanto para os trabalhadores, pois a exaustão, o cansaço e a incapacidade de atender a todos com o mesmo critério de qualidade causam a insatisfação de ambos os atores envolvidos no processo do acolhimento. Os sujeitos envolvidos na prática necessitam de respaldo para uma boa atuação.

Em estudo realizado em Salvador/BA, foram identificadas estratégias para a melhoria da acessibilidade e da humanização do acolhimento: implantação de sistemas de marcação de consulta (marcação permanente, marcação de consulta por telefone, criação de listas de esperas); criação de equipes de acolhimento multiprofissional; avaliação de prioridades; orientação de fluxos; e educação permanente da equipe, com oficinas de sensibilização (VIEIRA-DA-SILVA ET AL. 2010).

Em estudo de Baraldi e Souto (2011), os resultados demonstraram que a grande quantidade de demanda não estava relacionada à falta de vagas, mas à desestruturação de um planejamento de ações. A concepção de que a formação das filas e o longo tempo de espera são características culturais da população tem sido contestada. Desta forma, fica evidente que, quando o serviço organiza estratégias para a facilitação do acesso do usuário com iniciativas criativas, é possível aumentar a satisfação destes, com consequente melhoria do cuidado prestado.

Baraldi e Souto apud Santana (2012) colocam que estratégias baseadas em tentativa e erro não são capazes de garantir a qualidade do serviço, tampouco proporcionar satisfação à clientela, ou harmonia e interação saudável entre os profissionais.

Estudos apontam que o acolhimento como um dispositivo de humanização tem a potencialidade de reduzir a demanda reprimida, oferecendo maior acesso aos serviços e responsabilizando toda a equipe pelo cuidado e pela satisfação do usuário. Além disso, permite desencadear um processo de mudança nas práticas de saúde (TAKEMOTO; SILVA, 2007; OLIVEIRA; TUNIN; SILVA, 2008; SCHOLZE; DUARTE-JUNIOR; SILVA, 2009; SANTANA ET AL., 2012).

Ressalvas existem quanto a essa reordenação das práticas assistenciais, de modo que essa descentralização do sujeito médico como protagonista do cuidado ainda precisa ser sensibilizada para os usuários que não aceitam completamente o papel da equipe multidisciplinar. Se realizado de forma desestruturada, pode criar conflitos e dificultar os esforços de comunicação, em especial, pela desvalorização da sua atividade, com possível sobrecarga aos profissionais em ambientes onde não haja um preparo tecnológico para lidar com esses impasses. Isto, apesar de experiências positivas demonstrarem boa aceitação na interdisciplinaridade e estarem em consonância com os objetivos do acolhimento e da PNH.

\section{Percepção dos profissionais}

Foram selecionados, para esta discussão, 7 artigos (21\%). Dentre os discursos mais abordados nos textos, destacam-se cinco temáticas relevantes: 'Significado e concepção dos profissionais'; 'Profissional que realiza o acolhimento'; 'Características e competências'; 'Fatores facilitadores para o acolhimento'; e 'Fatores que dificultam o acolhimento'. 
Para os profissionais, o acolhimento significa uma prática de recepção do usuário, através de atitudes e comportamentos atenciosos; é dar uma atenção especial e levar em conta uma assistência integral e holística, de modo que exista uma responsabilização do cuidado, sendo pela resolubilidade dos problemas ou pelos encaminhamentos necessários.

Entretanto, ainda podem-se perceber falas de profissionais que acreditam que o acolhimento está relacionado à prática da triagem, na qual se seleciona, encaminha, direciona ou passa o usuário adiante, ou que também pode ser interpretada como uma forma de agilizar o processo de trabalho e possibilitar o acesso desse mesmo usuário (FREIRE ET AL., 2008; JUNGES ET AL., 2012).

Para Pinheiro e Oliveira (2011), essa percepção, por parte dos profissionais, causa um 'empobrecimento' do sentido mais amplo do que significa o acolhimento, ou seja, um dispositivo tecno-assistencial de organização do processo de trabalho.

Em estudo realizado com profissionais de unidades de saúde do Nordeste, estes relataram que o acolhimento se apresenta como um elemento importante e tem contribuído para a organização do processo de trabalho e da demanda, e, apesar das dificuldades e dos entraves, foi capaz de produzir, na equipe, uma compreensão mais ampla das necessidades de saúde dos usuários, que transpõem o conceito saúde-doença (SOUZA ET AL., 2008).

Estes conceitos dispostos pelos profissionais estão em conformidade com a $\mathrm{PNH}$, onde o conceito de acolhimento está pautado na recepção do usuário, desde sua entrada no sistema, de forma a responsabilizar-se integralmente por ele, através de escuta qualificada, de forma que o indivíduo possa expressar suas queixas e preocupações (BRASIL, 2006). Propõe-se, ainda, servir de elo entre a necessidade dos usuários e as várias possibilidades de cuidado (TESSER; POLI-NETO; CAMPOS, 2010). É um recurso destinado a apoiar a qualificação do sistema de saúde, pois possibilita ao usuário o acesso a um cuidado justo, ampliado e integral, a partir do reconhecimento de que esse acesso é um direito humano fundamental (BARALDI; SOUTO, 2011).

Por meio da análise dos textos selecionados, os principais profissionais citados no processo de acolhimento foram: auxiliar/ técnico de enfermagem, gerente/gestor, enfermeiro, médico, cirurgião-dentista e outros profissionais não ligados à saúde.

Em estudo de Freire et al. (2008), os profissionais da enfermagem (auxiliares e enfermeiros) foram apontados como os profissionais mais bem preparados para realizar o acolhimento. A realização do acolhimento não deve estar restrita somente a um profissional de saúde. Toda a equipe deve estar envolvida no processo de acolher, pois todo profissional deve estabelecer uma relação com o usuário.

Em conformidade a este processo foram elencadas algumas características necessárias ou desejáveis para a prática dessa ação, tais como: capacidade de escuta; empatia; bom relacionamento com a população; paciência; e afetividade. Outro ponto evidenciado na análise diz respeito ao preparo técnico-científico que fundamenta a ação, bem como ao embasamento dos aspectos legais referentes ao exercício profissional, em que a equipe deve desempenhar as atribuições pertinentes à sua categoria profissional (FREIRE ET AL., 2008).

Dentre os fatores que facilitam e dificultam a prática do acolhimento (quadro 1), pode-se observar que foram apontados inúmeros entraves para a realização do acolhimento, na opinião dos profissionais. Para Oliveira, Tunin e Silva (2008), o acolhimento tem se constituído em um permanente desafio, repleto de tensões. Para os autores, é necessário investir em formas de receber os diferentes modos através dos quais o usuário busca ajuda, respeitando cada um, com suas especificidades e demandas. 


\begin{tabular}{|c|c|}
\hline & Facilitadores \\
\hline Receptividade da comunidade & FREIRE et al., 2008 \\
\hline $\begin{array}{l}\text { Espaços de conversação entre profissionais e } \\
\text { usuários }\end{array}$ & $\begin{array}{l}\text { CARDOSO et al., 2009; CAVALCANTE FILHO et al., 2009; } \\
\text { PINHEIRO; OLIVEIRA, } 2011\end{array}$ \\
\hline \multirow[t]{2}{*}{ Maior visibilidade ao trabalho dos profissionais } & CAVALCANTE FILHO et al., 2009 \\
\hline & Dificultadores \\
\hline Falta de espaço físico adequado & $\begin{array}{l}\text { FREIRE et al., 2008; NERY et al., 2009; MATUMOTO et al., 2009; } \\
\text { BREHMER; VERDI, 2010; DAMASCENO et al., } 2012\end{array}$ \\
\hline Falta de privacidade & $\begin{array}{l}\text { FREIRE et al., 2008; NERY et al., 2009; MATUMOTO et al., 2009; } \\
\text { BREHMER; VERDI, 2010; DAMASCENO et al., } 2012\end{array}$ \\
\hline $\begin{array}{l}\text { Não aceitação do acolhimento/Resistência dos } \\
\text { usuários }\end{array}$ & FREIRE et al., 2008; JUNGES et al., 2012 \\
\hline Falta de valorização e reconhecimento & PINAFO; LIMA; BADUY, 2008; NERY et al., 2009 \\
\hline $\begin{array}{l}\text { Conciliação das atividades da unidade com } \\
\text { atividades externas (visita domiciliar) }\end{array}$ & NERY et al., 2009; PINHEIRO; OLIVEIRA, 2011 \\
\hline Falta de funcionários de outras áreas para apoio & NERY et al., 2009 \\
\hline Falta de profissionais & NERY et al., 2009; BREHMER; VERDI, 2010; OLIVEIRA et al., 2010 \\
\hline Sobrecarga de trabalho & NERY et al., 2009; PINHEIRO; OLIVEIRA, 2011 \\
\hline $\begin{array}{l}\text { Organização das reuniões de avaliação das ações } \\
\text { das equipes }\end{array}$ & NERY et al., 2009 \\
\hline Falta de preparo dos profissionais & OLIVEIRA et al., 2010 \\
\hline $\begin{array}{l}\text { Pressão da demanda, que gera um tempo } \\
\text { reduzido para o atendimento }\end{array}$ & NERY et al., 2009; COELHO; JORGE, 2009 \\
\hline Atividades burocráticas & PINAFO; LIMA; BADUY, 2008; MATUMOTO et al., 2009 \\
\hline Infraestrutura e falta de recursos & PINHEIRO; OLIVEIRA, 2011; COELHO; JORGE, 2009 \\
\hline Falta de limpeza & MATUMOTO et al., 2009 \\
\hline Sensação de impotência & JUNGES et al., 2012 \\
\hline Falta de comunicação & COELHO; JORGE, 2009 \\
\hline
\end{tabular}

Fonte: Elaboração própria

\section{Percepção dos usuários}

A partir da análise de 9 artigos (27\%), puderam-se sistematizar três temáticas predominantes, nas falas dos usuários: 'Conhecimento sobre o acolhimento'; 'Percepção/sentimento'; e 'Acessibilidade no acolhimento’.

Através da análise das percepções dos usuários pode-se constatar que estes relacionam o acolhimento à boa receptividade (bom atendimento) pela equipe, ao direcionamento para consultas médicas, ao fornecimento de medicamentos, à resolução dos problemas de saúde, à triagem (avaliação de sinais vitais e classificação de prioridades) e, ainda, relacionam o acolhimento ao atendimento executado pelo profissional médico ou enfermeiro.

Em pesquisa produzida por Santana et al. (2012), observou-se que a percepção do usuário sobre o que seria o acolhimento estava relacionada ao nível de instrução e aos aspectos sociais e culturais dos usuários, bem como às suas expectativas quanto a serem atendidos e terem seus problemas resolvidos.

Quanto ao sentimento/percepção dos usuários sobre o acolhimento, o que pôde ser encontrado foram atributos que ocasionavam satisfação ou insatisfação (quadro 2). 
Quadro 2. Sentimentos/percepções dos usuários que ocasionam satisfação e insatisfação quanto ao acolhimento

\begin{tabular}{|c|c|}
\hline \multicolumn{2}{|c|}{ Satisfação } \\
\hline Bom atendimento & $\begin{array}{l}\text { PINAFO; LIMA; BADUY, 2008; MACEDO; TEIXEIRA; } \\
\text { DAHER, 2011; SANTANA et al., } 2012\end{array}$ \\
\hline Escuta às queixas & COELHO; JORGE; ARAUJO, 2009; SANTANA et al., 2012 \\
\hline Presença de instrumentos que favorecem o atendimento & LIMA et al., 2007; COELHO; JORGE; ARAUJO, 2009 \\
\hline Ambiência & $\begin{array}{l}\text { PINAFO; LIMA; BADUY, 2008; COELHO; JORGE; } \\
\text { ARAUJO, } 2009\end{array}$ \\
\hline Responsabilização e comprometimento dos profissionais & PINAFO; LIMA; BADUY, 2008 \\
\hline Resolução dos problemas & MARTHA et al., 2011 \\
\hline \multicolumn{2}{|c|}{ Insatisfação } \\
\hline Falta do profissional médico & SANTANA et al., 2012 \\
\hline Filas e longa espera & $\begin{array}{l}\text { LIMA et al., 2007; MARTHA et al., 2011; SANTANA et al., } \\
2012\end{array}$ \\
\hline Falta de recursos materiais e humanos & SANTANA et al., 2012; MARTHA et al., 2011 \\
\hline Estrutura física inadequada & COELHO; JORGE; ARAUJO, 2009; MARTHA et al., 2011 \\
\hline Morosidade na marcação e no resultado de exames & SANTANA et al., 2012 \\
\hline Impessoalidade na relação entre profissional e usuário & COELHO; JORGE; ARAUJO, 2009 \\
\hline Dificuldade de acesso a atendimento especializado & $\begin{array}{l}\text { PINAFO; LIMA; BADUY, 2008; COELHO; JORGE; } \\
\text { ARAUJO, 2009; MARTHA et al., } 2011\end{array}$ \\
\hline Falta de privacidade & COELHO; JORGE; ARAUJO, 2009 \\
\hline Falta de preparo dos profissionais & COELHO; JORGE; ARAUJO, 2009 \\
\hline Falta de respeito & OLIVEIRA et al., 2008; MARTHA et al., 2011 \\
\hline
\end{tabular}

Fonte: Elaboração própria

Ao acolhimento, para Brehmer e Verdi (2010), é atribuído também o significado de acesso aos serviços de saúde, sendo considerado como a porta de entrada prioritária para o usuário.

Quanto à acessibilidade no acolhimento, pode-se observar que os usuários percebem o acesso como oferecimento de ações e serviços, recepção administrativa eficaz e estabelecimento de vínculo com os profissionais.

Savassi (2010) coloca que a percepção do acesso aos serviços de saúde está relacionada ao espaço geográfico, ou seja, se dá pela adscrição da clientela e a boa relação com os profissionais, que, por sua vez, estão relacionadas ao nível de satisfação da população com o serviço. A percepção do usuário e sua satisfação com o serviço interferem na acessibilidade e na criação de vínculos, o que pode provocar dificuldades, para a equipe, de coordenar o cuidado, refletindo na qualidade do serviço. Lima et al. (2007) reforçam este conceito, de que o vínculo, a resolutividade e o desempenho profissional estão relacionados ao acolhimento.

\section{Conclusões}

Mediante o subsídio dos artigos analisados, pode-se afirmar que o processo de acolhimento ainda não está totalmente sistematizado nos modelos de atenção à saúde, podendo ser esta uma justificativa para as dificuldades apresentadas, tanto por profissionais quanto por usuários.

O acolhimento é um método designado para colaborar com a qualificação dos sistemas de saúde, de forma que possibilite ao usuário o acesso a um atendimento justo e integral, por meio da multiprofissionalidade e da intersetorialidade. É uma ferramenta capaz de possibilitar que o SUS efetive seus princípios constitucionais. 
Entretanto, ainda se faz necessário qualificar a maneira como vem sendo desenvolvido, pois, se trabalhado de forma desarticulada e pontual, pode ser resumido a uma mera atividade de triagem e descaracterizado de sua principal função: a humanização. Sendo assim, não alcançaria seus propósitos, caso fosse implantado como medida isolada.

Pela análise dos textos, fica evidente que não existe uma uniformidade quanto à prática do acolhimento. Mesmo levando-se em consideração que cada serviço tem suas peculiaridades e individualidades, é necessária certa forma de organizar o serviço, que deverá ser pautado em normatizações e, principalmente, em incentivos à educação permanente. $\mathrm{O}$ serviço é que precisa se organizar para oferecer o acolhimento, e não o contrário.

Uma consideração quanto à percepção de profissionais e usuários é a de que os benefícios da prática acolhedora acabam ficando sem a devida relevância quando se observa que existem mais obstáculos para a realização dessa atividade do que pontos positivos e estimuladores da mesma. É evidente que ambientes desfavoráveis, e tantos outros pontos apontados nas análises são dificultadores, porém o ato de humanização ultrapassa as atividades rotineiras e deve ser encarado como algo intrínseco à prática profissional.

São necessários estudos com novas abordagens ou estratégias para a sistematização do acolhimento nas unidades de APS, ou ainda, é preciso que sejam divulgadas as experiências positivas na prática dos profissionais, e se estas têm realmente impacto na qualidade dos serviços e na satisfação dos usuários, entendendo que esta é a 'peça-chave' de toda a organização do serviço.

\section{Referências}

\author{
ABRAHÃO, A. L. Atenção primária e o processo de \\ trabalho em saúde. Informe-se em promoção da saúde, \\ Niterói, v. 3, n. 1, p. 1-3, 2007. \\ BARALDI, D. C.; SOUTO, B. G. A. A demanda do aco- \\ lhimento em uma unidade de saúde da família em São \\ Carlos, São Paulo. Arq. Bras. Cienc. Saúde, Santo André, \\ v. 36, n. 1, p. 10-17, 2011.
}

BRASIL. Ministério da Saúde. HumanizaSUS: documento base para gestores e trabalhadores do SUS. 3. ed. Brasília, DF: Ministério da Saúde, 2006.

Ministério da Saúde. Secretaria de Atenção à

Saúde. Departamento de Atenção Básica. Acolhimento à demanda espontânea. Brasília: Ministério da Saúde, 2011.

Ministério da Saúde. Secretaria de Atenção

à Saúde. Núcleo Técnico da Política Nacional de
Humanização. Acolhimento nas práticas de produção de saúde. 2. ed. Brasília, DF: Ministério da Saúde, 2010.

BREHMER, L. C. F; VERDI, M. Acolhimento na Atenção Básica: reflexões éticas sobre a Atenção à Saúde dos usuários. Ciência \&́ Saúde Coletiva, Rio de Janeiro, v. 15, supl. 3, p. 3569-3578, 2010.

CARDOSO, L. S. C. et al. Acolhimento no trabalho em saúde da família: um estudo qualitativo. CuidArte Enfermagem, Juiz de Fora, v. 3, n. 2, p. 149-155, 2009.

CARVALHO, C. A. P. et al. Acolhimento aos usuários: uma revisão sistemática do atendimento no Sistema Único de Saúde. Arq. Ciênc. Saúde, São José do Rio Preto, v. 15, n. 2, p. 93-98, 2008.

CAVALCANTE FILHO, J. B. et al. Collective welcoming: a challenge instigating new ways of producing 
care. Interface (Botucatu), Botucatu, v. 13, n. 31, p. 315$328,2009$.

COELHO, M. O.; JORGE, M. S. B. Tecnologia das relações como dispositivo do atendimento humanizado na atenção básica à saúde na perspectiva do acesso, do acolhimento e do vínculo. Ciência \&t Saúde Coletiva, Rio de Janeiro, v. 14, supl. 1, p. 1523-1531, 2009.

COELHO, M. O.; JORGE, M. S. B.; ARAUJO, M. E. O acesso por meio do acolhimento na Atenção Básica à Saúde. Revista Baiana de Saúde Pública, Salvador, v. 33, n. 3, p. 440-452, 2009.

DAMASCENO, R. F. et al. O acolhimento no contexto da Estratégia Saúde da Família. J Health Sci Inst, São Paulo, v. 30, n. 1, p. 37-40, 2012.

FREIRE, L. A. M. et al. O acolhimento sob a ótica de profissionais da equipe de Saúde da Família. Reme, Belo Horizonte, v. 12, n. 2, p. 271-277, 2008.

HENNINGTON, E. A. Acolhimento como prática interdisciplinar num programa de extensão universitária. Cadernos de Saúde Pública, Rio de Janeiro, v. 21, p. 256-265, 2005.

JUNGES, J. R. et al. O discurso dos profissionais sobre demanda e a humanização. Saúde soc., São Paulo, v. 21, n. 3, p. 686-697, 2012.

LIMA, A. S. O trabalho da enfermeira na Atenção Básica: uma revisão sistemática. 2011. 134 f. Dissertação (Mestrado em Enfermagem) - Universidade Federal da Bahia, Salvador, 2011.

LIMA, M. A. D. S. et al. Acesso e acolhimento em unidades de saúde na visão dos usuários. Acta Paul Enferm, São Paulo, v. 20, n. 1, p. 12-17, 2007.

\section{MACEDO, C. A.; TEIXEIRA, E. R.; DAHER, D. V.}

Possibilidades e limites do acolhimento na percepção de usuários. Rev. Enferm. UERJ, Rio de Janeiro, v. 19, n. 3, p. 457-462, 2011.

MARTHA, S. N. et al. Programa Saúde da Família sob a visão do usuário. Salusvita, Bauru, v. 30, n. 3, p. 159-177, 2011.
MATUMOTO, S. et al. Preparando a relação de atendimento: ferramenta par o acolhimento em unidades de saúde. Rev. Latino-Am Enfermagem, Ribeirão Preto, v. 17, n. 6, p. 1001-1008, 2009.

MENDES, K. D. S.; SILVEIRA, R. C. C. P.; GALVÃO, C. M. Revisão Integrativa: métodos de pesquisa para a incorporação de evidências na saúde e na enfermagem. Texto Contexto Enfermagem, Florianópolis, v. 17, n. 4, p. 758-764, 2008.

MENDES, E. V. 25 anos do Sistema Único de Saúde: resultados e desafios. Estudos Avançados, São Paulo, v. 27, n. 78, p. 27-34, 2013.

NERY, S.R. et al. Acolhimento no cotidiano dos auxiliares de enfermagem nas Unidades de Saúde da Família, Londrina (PR). Ciência \&t Saúde Coletiva, Rio de Janeiro, v. 14, supl. 1, p. 1411-1419, 2009.

OLIVEIRA, E. R. A. et al. Acolhimento em saúde e desafios em sua implementação: percepção do acadêmico de enfermagem. Revista Brasileira de Pesquisa em Saúde, Vitória, v. 12, n. 2, p. 46-51, 2010.

OLIVEIRA, L. M. L.; TUNIN, A. S. M.; SILVA, F. C. Acolhimento: concepções, implicações no processo de trabalho e na atenção em saúde. Rev. APS, Juiz de Fora, v. 11, n. 4, p. 362-37, 2008.

OLIVEIRA, A. et al. A comunicação no contexto do acolhimento em uma unidade de saúde da família de São Carlos, SP. Interface (Botucatu), Botucatu, v. 12, n. 27, p. 749-762, 2008.

\section{ORGANIZAÇÃO PAN-AMERICANA DA SAÚDE} (OPAS). Renovação da Atenção Primária em Saúde nas Américas: documento de posicionamento da OPAS/ OMS. Washington, DC: OPAS, 2007.

PINAFO, E.; LIMA, J. V. C.; BADUY, R. S. Acolhimento: concepção dos auxiliares de enfermagem e percepção de usuários em uma unidade de saúde da família. Revista Espaço para Saúde, Londrina, v. 9, n. 2, p. 17-25, 2008.

PINHEIRO, P. M.; OLIVEIRA, L. C. A contribuição do acolhimento e do vínculo na humanização da 
prática do cirurgião-dentista no Programa Saúde da Família. Interface (Botucatu), Botucatu, v. 15, n. 36, p. 187-198, 2011.

SANTANA, J. C. et al. Acolhimento em um serviço da Atenção Básica à Saúde de Minas Gerais. Revista de Enfermagem do Centro Oeste Mineiro, São João del-Rei, v. 2, n. 2, p. 166-176, 2012

SANTOS, S. A. S. et al. The domiciliary visit as a practice of reception in the medical program of Family/ Niterói. R. Pesq.: Cuid. Fundam. Online, Rio de Janeiro, v. 5 , n. 2 , p. $3698-3705,2013$.

SAVASSI, L. C. M. A satisfação do usuário e a autopercepção da saúde em atenção primária [Editorial]. Revista Brasileira de Medicina de Família e Comunidade, Rio de Janeiro, v. 5, n. 17, p. 3-5, 2010.

SCHOLZE, A. S.; DUARTE JUNIOR, C. F.; FLORES E SILVA, Y. Trabalho em saúde e a implantação do acolhimento na atenção primária à saúde: afeto, empatia ou alteridade? Interface (Botucatu), Botucatu, v. 13, n. 31, p. 303-14, 2009.

SOUZA, E. C. F. et al. Acesso e acolhimento na atenção básica: uma análise da percepção dos usuários e profissionais de saúde. Cadernos de Saúde Pública, Rio de Janeiro, v. 24, supl. 1, p. s100-s110, 2008.

SOUZA, M. T.; SILVA, M. D.; CARVALHO, R. Revisão Integrativa: o que é e como fazer. Einstein, São Paulo, v. 8, n. 1, pt. 1, p. 102-106, 2010.
STARFIELD, B. Atenção primária: equilíbrio entre necessidades de saúde, serviços e tecnologia. Brasília: UNESCO: Ministério da Saúde, 2002.

TAKEMOTO, M. L. S.; SILVA, E. M. Acolhimento e transformações no processo de trabalho de enfermagem em unidades básicas de saúde de Campinas, São Paulo, Brasil. Cadernos de Saúde Pública, Rio de Janeiro, v. 23, n. 2, p. 331-340, 2007.

TESSER, C. D.; POLI-NETO, P.; CAMPOS, G. W. S. Acolhimento e (des)medicalização social: um desafio para as equipes de saúde da família. Ciência \& Saúde Coletiva, Rio de Janeiro, v. 15, supl. 3, p. 3615-624, 2010.

VIEIRA-DA-SILVA, L. M. et al. Avaliação da implantação de programa voltado para melhoria da acessibilidade e humanização do acolhimento aos usuários na rede básica. Salvador, 2005-2008. Rev. Bras. Saúde MaternoInfant, Recife, v. 10, supl. 1, p. s131-143, 2010.

WORLD HEALTH ORGANIZATION (WHO). The Word Health Report 2008: Primary Health Care now more than ever. Geneva: WHO, 2008.

Recebido para publicação em julho de 2014

Versão final em fevereiro de 2015

Conflito de interesses: inexistente

Suporte financeiro: não houve 